\title{
Preocupante incremento de la diabetes como causa de insuficiencia renal terminal. Evaluación del tratamiento sustitutivo
}

\author{
R. PÉREZ GARCÍA, P. RODRÍGUEZ BENÍTEZ, C. DALL'ANESSE, F. GÓMEZ \\ CAMPDERÁ, F. VALDERRÁBANO.
}

Servicio de Nefrología. Hospital General Universitario “Gregorio Marañón”. Madrid.

\author{
INCREASING INCIDENCE OF RENAL REPLACEMENT THERAPY IN \\ DIABETIC PATIENTS
}

\section{RESUMEN}

Objetivo: Valorar la incidencia, prevalencia, tipo de tratamiento y pronóstico de pacientes diabéticos en tratamiento sustitutivo de la función renal (TSFR).

Material: Se estudia a todos los pacientes diabéticos con insuficiencia renal terminal en TSFR en el Área Sanitaria 1 de Madrid desde 1978 hasta 1998.

Resultados: La diabetes mellitus se ha convertido en la principal causa de insuficiencia renal terminal en nuestra área sanitaria, constituyendo en la actualidad el 30\% de todas las causas de entrada en TSFR. La incidencia de diabéticos que comenzaron en 1998 TSFR fue de 33,3 pmp, claramente superior a la del periodo 1993-94, 21 pmp. La prevalencia de pacientes diabéticos en TSFR en 1998 fue de 135 pmp, con una prevalencia global de $1054 \mathrm{pmp}$. Se ha incrementado la proporción de diabéticos tipo 2 con respecto al global de pacientes diabéticos que inician tratamiento sustitutivo, pasando de ser un $15 \%$ en $1987-88$ a un $81 \%$ en 1997-98. De manera simultánea, se ha incrementado la edad media de dichos pacientes, pasando de ser 47 años antes de 1988 a 63 años en 1997-98. En total se estudian 182 diabéticos, 106 hombres y 76 mujeres. 105 eran diabéticos tipo 1 y 77 tipo 2 , con una edad media al comienzo del TSFR de 5712 años. La hemodiálisis (HD) fue la primera modalidad de tratamiento en 128 pacientes y la diálisis peritoneal (DP) en 54. Los pacientes que iniciaban DP eran 5 años más jóvenes que los que iniciaban HD, p $<0,01$, con una mayor proporción de diabéticos tipo $1,72 \%$ en DP frente a un $51 \%$ en HD, p $<0,05$.

El periodo de seguimiento fue 5145 meses y durante éste, 79 pacientes cambiaron su modalidad de tratamiento y 45 de los cuales recibieron un trasplante renal (TxR). Las principales complicaciones observadas fueron las oftalmológicas, $77 \%$, seguidas de la vasculopatía periférica con un $35 \%$ de casos (10\% de amputados). Los accidentes cerebrovasculares y el infarto agudo de miocardio acontecieron en un 21 y $17 \%$ de casos respectivamente. A lo largo del periodo de estudio, murieron 89 pacientes. Las enfermedades cerebro y cardiovasculares constituyeron la causa más frecuente (29\%), seguida de las infecciones (27\%). La supervivencia total fue de $92,80,72,61$ y $54 \%$ en el $1,2,3,4$ y 5 años respectivamente. La supervivencia fue mejor en HD que en DP, aunque sin alcanzar significación estadística. La menor edad y el TxR eran variables con efecto independiente positivo en la supervivencia, mientras que la vasculopatía distal tenía un importante efecto negativo.

Conclusiones: Se comprueba un alarmante aumento en la incidencia de diabéticos tipo 2 que inician TSF. El TxR es la técnica con mejores resultados.

\section{ABSTRACT}

Objective: To study the incidence, prevalence, treatment modalities and prognosis of diabetic patients on renal replacement therapy.

Material: We studied all end-stage renal failure (ESRF) diabetic patients on renal replacement therapy in "Area Sanitaria 1" in Madrid from 1978 to 1998.

Results: Diabetes mellitus has become the leading cause of ESRF in our health area of Madrid, $30 \%$ of all causes of ESRF. Incidence of diabe tics beginning RRT was 33,3 per million population ( pmp) in 1998, while in 1993-94 diabetes was $15 \%$ and 21 pmp, respectively, table 1. The propor tion of diabetics on RRT has increased from 7,4 \% 1986 to 12,7\% 1998. The prevalence of diabetics on RRT in 1998 was $135 \mathrm{pmp}$, with an overall prevalence of $1054 \mathrm{pmp}$. At the mean time, the proportion between incident diabetics type 2/ diabetics on RRT has increased from $15 \%$ in 1987-88, to $54 \%$ in 1993-94 and to $81 \%$ in 1997-98, consequently, mean age of diabe tic patients at beginning RRT has increased from 47 years before 1988, 58 in 1989-90, 61 in 1993-94, 62 in 1995-96 and 63 in 1997-98 (Table I).

We studied 182 diabetics admitted for renal replacement therapy, 106 males and 76 females, 105 were diabetics type 1 and 77 type 2. Their mean age at RRT beginning was 5712 years (SD). Hemodialysis (HD) was the first modality of treatment for 128 (70\%) diabetics and CAPD for 54. Seventy out of 128 patients on HD were dialyzed in the Hospital Unit, $40 \%$ on AFB (acetate free biofiltration) and 58 in two Satellite Units, that means a higher proportion of diabetics on CAPD and on $H D$ in Hospital Unit. Diabetics allocated to CAPD were 5 years, as mean, younger than patients allocated to $H D(p<0.01)$ and the proportion of diabetes type 1 was higher in CAPD (72\%) than in $H D(51 \%), p 0,05$.

During the mean follow-up period (51 45 months) 79 patients chan ged their treatment modality and 45 of them received a kidney allogra[. Relative risk of drop-out was higher in CAPD technique when compared to HD. Clinical complications were frequently observed: ocular (77\%), cardiovascular (Myocardial infarction $17 \%$ ), acute cerebrovascular disease $(21 \%)$ and distal angiopathy $(35 \%), 10 \%$ amputee.

At December of 1998, 89 patients were dead. Cardiovascular and cerebrovascular diseases (29\%) and Infections (27\%) were the two most frequent causes of death. Uknown-origin deaths represent $19 \%$ of all deaths. The overall survival (Kaplan Meier) was 92\%, 80\%, 72\%, 61\% and $54 \%$ at 1,2, 3, 4 and 5 years, respectively (57 patients completed last period). Survival was better on HD than on CAPD, but without statistical significance, although the significant difference in age and in proportion of diabetes type 2 between both groups. Data analysis estima ted by Cox proportional hazards regression showd that younger age and ki,r transplantation had a positive independent effect on survival, whik clinical distal angiopathy had important negative effect on survival.

Conclusions: Diabetes mellitus has become the leading case of ESRF in our health area of Madrid. Young age and kidney transplanta tion had a positive independent effect on survival, while clinical distal angiopathy had important negative effect. 
PALABRAS CLAVE: Diabetes. Tratamiento sustitutivo de la función renal. Diálisis. Trasplante renal. Epidemiología. Mortalidad.
KEY WORDS: Diabetes. Renal replacement therapy. Peritoneal dialy sis. Transplantation. Epidemiology. Mortality.

Pérez García R, Rodríguez Benítez P, Dall'Anesse C, Gómez Campderá F, Valderrábano F. Preocupante incremento de la diabetes como cau sa de insuficiencia renal terminal. Evaluación del tratamiento sustitutivo. An Med Interna (Madrid) 2001; 18: 175-180.

\section{INTRODUCCIÓN}

En los últimos años, se ha producido un aumento tanto en el número de pacientes incluidos en programa de tratamiento sustitutivo de la función renal (TSFR) como en la edad media de los mismos (1-4). En España, como en el resto de Europa, hemos asistido a un incremento progresivo del número de diabéticos que son incluidos en TSFR (5-8). La diabetes, fundamentalmente la tipo 2, representa una proporción cada vez mayor del total de causas que condicionan la entrada en un programa de diálisis periódicas (5-8). Varias causas pueden explicar este hecho: en primer lugar, el mejor tratamiento de las complicaciones de la diabetes, con el consiguiente aumento de su supervivencia y la mayor probabilidad de desarrollar nefropatía progresiva y precisar TSFR; en segundo lugar, somos menos selectivos a la hora de incluir a un paciente en TSFR y la edad o la enfermedad cardiovascular concomitante han dejado de ser criterios de exclusión. No debemos olvidar que hasta hace poco tiempo existían servicios de nefrología que no consideraban a los diabéticos, debido a su mal pronóstico y elevada morbilidad, como candidatos para ingresar en un programa TSFR. En la actualidad, el pronóstico de los diabéticos en TSFR ha mejorado, aunque sigue siendo peor que el del resto de enfermos con insuficiencia renal terminal (911).

Es controvertido establecer el TSFR de elección en este grupo de pacientes. Durante años, muchos nefrólogos apostaban por la diálisis peritoneal continua ambulatoria (DPCA) como el tratamiento sustitutivo de elección (12-14). En la actualidad, existen trabajos que obtienen mejores resultados con las nuevas técnicas de hemodiálisis-hemodiafiltración, y reivindican el papel beneficioso de dichos tratamientos (15). Los criterios de selección utilizados en los diabéticos para considerarlos candidatos a trasplante renal se han ampliado en los últimos años, aunque siguen sin estar totalmente claros $(6,16,17)$. De cualquier modo, el trasplante renal constituye, por lo general, el método con el que se obtiene una mayor supervivencia.

El objetivo fundamental de este trabajo fue valorar los resultados, de epidemiología y morbi-mortalidad, de los pacientes diabéticos incluidos en TSFR en nuestra área sanitaria. Objetivos secundarios fueron reevaluar la indicación del TSFR inicial y proponer nuevas estrategias terapéuticas en dichos pacientes. El alarmante aumento de la incidencia de pacientes incluidos en TSFR en nuestra Area de Madrid y la necesidad de ofrecer a los diabéticos un tratamiento integrado de la insuficiencia renal que incluya el trasplante renal, la hemodiálisis y la diálisis peritoneal, constituyen nuestras principales conclusiones.

\section{MATERIAL Y MÉTODOS}

En 1993, en el Servicio de Nefrología del HGU Gregorio Marañón, se creó un protocolo de recogida de datos sobre la morbi-mortalidad de los pacientes diabéticos en TSFR pertenecientes a nuestra área sanitaria. En ese mismo año y basándonos en dicho protocolo, se inició este trabajo, en el que se analizan los resultados de morbi-mortalidad de nuestros diabéticos en TSFR desde 1978, año en que se incluyó en un programa de TSFR al primer diabético, hasta Diciembre de 1998, momento en que se cierra el estudio. Se trata, por consiguiente, de un estudio retrospectivo desde 1978 hasta 1993 y prospectivo desde ese momento hasta su conclusión. Durante este periodo de tiempo se trataron en nuestro servicio 186 pacientes diabéticos, de los cuales se incluyeron en este estudio 182. Los cuatro restantes fueron excluidos al no encontrarse en su historia clínica todos los datos que exigía el protocolo.

\section{CARACTERÍSTICAS DEL ÁREA SANITARIA, HOSPITAL Y CENTROS SATÉLITES}

Nuestro Servicio de Nefrología, en funcionamiento desde el año 1969, da cobertura sanitaria al Área 1 de Madrid, con una población estimada de 630.000 habitantes. Sólo asiste a pacientes adultos, mayores de 16 años. Engloba una unidad de diálisis hospitalaria con diferentes técnicas de hemodiálisis, que en la actualidad trata a 89 pacientes, $13,4 \%$ del total. Asimismo, existen dos centros satélites de hemodiálisis donde se hemodializan 230 pacientes, el 34,6\%. Desde 1982, existe un programa de DPCA, con capacidad para DPI y DPA, con 28 pacientes en el momento del cierre del estudio, $4,2 \%$ y un programa de hemodiálisis domiciliaria que engloba ahora a dos pacientes, el $0,3 \%$. El programa de trasplante renal se inició en el año 1976, habiéndose efectuado 670 trasplantes, de los cuales permanecen funcionantes 315 , el $47,4 \%$ del total de pacientes en TSFR. De este modo, considerando todas las modalidades, el número total de pacientes que se encuentran en TSFR es de 664 a fecha de Diciembre de 1998.

El hospital dispone también de un Servicio de Nefrología infantil, no incluido en este estudio.

\section{MÉTODOS}

Se revisaron las historias clínicas de todos los pacientes diabéticos incluidos en TSFR durante el periodo de estudio. Se consideraron los siguientes datos: sexo, edad, tipo de dia- 
betes mellitus, tipo de tratamiento inicial, transferencia posterior a otros tipos de tratamiento, tiempo de seguimiento, complicaciones oftalmológicas, complicaciones cardiovasculares y causa de muerte.

Clasificación de diabetes mellitus: Se consideró diabetes mellitus tipo 1 la de aquellos pacientes diagnosticados antes de los 30 años de edad y que precisaron insulina en los 3 primeros meses desde el diagnóstico y diabetes mellitus tipo 2, la de aquellos que no cumplían dichos criterios.

Complicaciones asociadas a la diabetes:

- Las complicaciones oftalmológicas se clasificaron en: ceguera total, disminución severa de la visión, presencia de cataratas, intervención quirúrgica de cataratas, ausencia de problemas visuales.

- Las complicaciones cardiovasculares consideradas, fueron: accidente cerebro-vascular, angor, infarto agudo de miocardio, isquemia en las extremidades, claudicación intermitente, amputación de causa isquémica.

Modalidades terapéuticas utilizadas como TSFR: hemodiálisis hospitalaria (HDH); hemodiálisis en centros satélites (HDCS); hemodiálisis domiciliaria (HDD); diálisis peritoneal continua ambulatoria (DPCA); diálisis peritoneal nocturna con cicladora (DPA) y trasplante renal (Tx).

- Criterios de inclusión aplicados en las diferentes modalidades terapéuticas enumeradas: Normalmente, se incluyen en HDH a los pacientes de mayor edad y con mayor número de condiciones comórbidas, entre las que se engloban las cardiopatías, vasculopatía severa de miembros inferiores, amputaciones, ceguera o secuelas neurológicas importantes. En general, inician HDCS aquellos pacientes no considerados candidatos para diálisis peritoneal y que no cumplen los criterios antes expuestos. En este punto, habría que resaltar que, desde hace unos cinco años, las diferencias entre los pacientes en HDCS y los de HDH han disminuido.

Se consideran candidatos para DPCA/DPA aquellos diabéticos jóvenes, sin limitaciones físicas importantes, como cardiopatía o amputación de uno o más miembros y que no presenten ceguera o disminución importante de la visión.

Se consideran candidatos para Tx todos los pacientes en TSFR que no presenten ninguna contraindicación para la cirugía. El límite admisible de edad, establecido anteriormente en 65 años, ha sido ampliado en la actualidad a los 70 años en personas sin gran comorbilidad. Los pacientes con cardiopatía isquémica, deberán ser diagnosticados y tratados antes de su inclusión en lista de espera para trasplante. Se excluirán los pacientes con arteriosclerosis severa.

\section{ANÁLISIS ESTADÍSTICO}

Para el procesamiento y análisis de los datos, se utilizó el programa estadístico Rsigma Babel, Horus S.A. Para comparar proporciones se utilizó la prueba exacta de Fischer. Las curvas de supervivencia se calcularon con el método de Kaplan Meier y para su comparación se empleó la prueba de Log-Rank. Se aplicó un análisis de supervivencia con covariantes, según los modelos de regresión de Cox. Todos los resultados se expresaron como la media \pm una desviación estándar. Se consideraron significativas las probabilidades menores del 5\%, p menor de 0,05 .

\section{RESULTADOS}

Del total de los 186 diabéticos, tratados durante el periodo de estudio, en 182 fue posible recoger todos los datos requeridos en el protocolo. Estos 182 pacientes representan el 12,9\% del total de adultos que han sido incluidos en TSFR en nuestro hospital en dicho periodo de tiempo $(n=1412)$. Respecto al total de pacientes, el porcentaje de pacientes diabéticos que en

DATOS EPIDEM IO LÓ GICOS DE LOS PACIENTES DIABÉTICOS EN TRATAM IENTO SUSTITUTIVO DE LA FUNCIÓN RENAL (TSFR) EN EL ÁREA SANITARIA 1 DE MADRID (DIÁLISISY TRASPLANTE RENAL)

\begin{tabular}{lcccccccc}
\hline Años & $1983-84$ & $85-86$ & $87-88$ & $89-90$ & $91-92$ & $93-94$ & $95-96$ & $97-98$ \\
\hline $\begin{array}{l}\text { Incidencia } \\
\text { diabéticos } \\
\text { no/pmp/año }\end{array}$ & 10 & 6 & 16 & 16 & 15 & 21 & 25 & 32 \\
$\begin{array}{l}\text { Pacientes diabéticos } \\
\text { nuevos en TSFR como } \\
\text { porcentaje del total \% }\end{array}$ & 7 & 9 & 12 & 11 & 12 & 15 & 25 & 29 \\
$\begin{array}{l}\text { Diabéticos tipo 2 } \\
\text { como porcentaje } \\
\text { del total de diabéticos }\end{array}$ & 17 & 37 & 15 & 5 & 11 & 54 & 74 & 81 \\
$\begin{array}{l}\text { incidentes \% } \\
\begin{array}{l}\text { Edad media de los } \\
\text { diabéticos incidentes } \\
\text { años }\end{array}\end{array}$ & 46 & 53 & 53 & 58 & 51 & 61 & 62 & 63 \\
$\begin{array}{l}\text { Prevalencia de diabéticos } \\
\text { como porcentaje de la } \\
\text { prevalencia total \% }\end{array}$ & - & 7,4 & 8 & 9,6 & 9,8 & 10 & 11,5 & 12 \\
\hline
\end{tabular}


1998 comenzaron TSFR fue del 30\%, claramente superior al de los años previos (Tabla I). La incidencia de diabéticos que comenzaron en 1998 TSFR fue de 33,3 pmp, claramente superior a la del periodo 1993-94, 21 pmp. La prevalencia de pacientes diabéticos en TSFR en 1998 fue de 135 pmp, con una prevalencia global de $1054 \mathrm{pmp}$. Se ha incrementado la proporción de diabéticos tipo 2 con respecto al global de pacientes diabéticos que inician tratamiento sustitutivo, pasando de ser un $15 \%$ en $1987-88$ a un $81 \%$ en $1997-98$. De manera simultánea, se ha incrementado la edad media de dichos pacientes, pasando de ser 47 años antes de 1988, a 63 años en 1997-98. En la tabla I se aportan los datos de la incidencia y prevalencia de los pacientes diabéticos de nuestra área.

Ciento seis pacientes (58\%) eran hombres y 76 mujeres (42\%), con una edad media al inicio del tratamiento de $57 \pm$ 12 años. Ciento cinco pacientes padecían una diabetes tipo 1 $(58 \%)$ y 77 (42\%) diabetes tipo 2 . Los pacientes con diabetes tipo 1 eran significativamente más jóvenes que aquellos con diabetes tipo 2, 50,8 $\pm 12,3$ versus 64,4 $\pm 6,9$ años, $\mathrm{p}<0,001$. La proporción de diabéticos tipo 2, respecto al total en TSFR, ha aumentado en los últimos años, pasando del $15 \%$ en $1987-$ 88 , al $54 \%$ en $1993-94$ y $81 \%$ en $1997-98$.

En cuanto al tratamiento inicial, 128 pacientes $(70 \%)$ recibieron hemodiálisis,70 (38\%) HDH y 58 (32\%) HDCS. El segundo tipo más frecuente de tratamiento inicial fue la DPCA, con 54 pacientes (30\%). Ningún paciente recibió un Tx renal como primer tratamiento. En los diabéticos existe un claro predominio de la HDH y la DPCA, cuando se comparan con el resto de pacientes: $30 \%$ del total de los pacientes en HDH versus $49 \%$ en los diabéticos; 5,2\% en DPCA, frente al $30 \%$ de los diabéticos. Por el contrario, existe un menor porcentaje de diabéticos que inician HDCS, $21 \%$ frente al $64,8 \%$ de los pacientes no diabéticos, $\mathrm{p}<0,01$. La edad media al comienzo del tratamiento fue de $57 \pm 12$ años, mayor en los pacientes que comenzaron tratamiento sustitutivo con HD, 59 \pm 11 , que aquellos que comenzaron en DPCA, $52 \pm 13$, diferencia significativa, $\mathrm{p}<0,01$. No se encontraron diferencias respecto al sexo en la modalidad inicial de tratamiento. La proporción de diabéticos tipo 1 entre los pacientes que iniciaron DPCA fue del $72 \%$, claramente mayor que la del grupo de pacientes que iniciaron $\mathrm{HD}, 51 \%, \mathrm{p}<0,05 \%$.

A lo largo del estudio se produjeron 79 cambios de modalidad terapéutica. Nueve pacientes pasaron de HD a DPCA y 15 de DPCA a HD. Cuarenta y cinco se trasplantaron. Cinco fueron transferidos desde un Centro Satélite a la Unidad Hospitalaria y cuatro, al contrario. Por último, un paciente pasó de la Unidad Hospitalaria a Diálisis Domiciliaria.

En cuanto a las complicaciones, un $77 \%$ de los pacientes presentaron complicaciones oftalmológicas. De estos, 24 pacientes manifestaron ceguera total y 96, disminución importante de la agudeza visual. Dieciséis pacientes presentaron cataratas uni o bilaterales y en otros 24 , ya existía, en el momento de incluirse en el estudio, el antecedente de cirugía de cataratas. Los pacientes en DPCA presentaban amaurosis en una proporción menor que los de HD, lo cual estaba en relación con el sistema de selección utilizado para la inclusión de los pacientes en las dos modalidades terapéuticas. No se encontró ninguna relación significativa entre la aparición de complicaciones oftalmológicas y la modalidad de tratamiento. Con respecto a las complicaciones cardiovasculares, el $21 \%$ de los pacientes había presentado un ACVA no mortal y el $17 \%$ había sufrido un IAM no letal. Finalmente, el 35\% de los pacientes presentaban síntomas o signos de isquemia en miembros inferiores y un $10 \%$ habían sufrido la amputación de al menos un miembro a lo largo de la evolución.

El tiempo medio de seguimiento fue de $51 \pm 45$ meses $(1-$ 229). Desglosado según la modalidad terapéutica: en HD $37 \pm$ 36 meses, en DPCA $25 \pm 19$ meses y en trasplante $55 \pm 45$ meses. De los 182 pacientes estudiados, se produjeron 8 pérdidas de seguimiento (4\%) y fallecieron 89 (49\%), encontrándose activos al cierre del estudio 85 pacientes (47\%). Las causas de muerte más frecuentes fueron las vasculares (IAM y ACVA ) $29 \%$, seguidas de las infecciones, $27 \%$ y de las origen desconocido, $19 \%$. En el momento de fallecer, 54 pacientes $(61 \%)$ se encontraban en HD, 24 en DPCA (27\%) y 11 eran portadores de un injerto renal funcionante $(12 \%)$. La supervivencia actuarial global fue del $92 \%$ al primer año, del $72 \%$ a los tres años y del 54\% a los cinco, existiendo un total de 57 pacientes activos si consideramos este último periodo de seguimiento. Los pacientes que fallecieron tenían una edad media significativamente mayor que los que sobrevivieron, $\mathrm{p}<0.05$. Cuando se compararon las supervivencias según el sexo y el tratamiento inicial no se encontraron diferencias significativas. Los pacientes que recibieron un Tx renal tuvieron una supervivencia actuarial significativamente mejor que los que no se trasplantaron, con una supervivencia del $98 \%$ en el primer año, $91 \%$ a los 3 años y $81 \%$ a los 5 años, (Log-Rank $\mathrm{p}<0,01)$. Comparando la supervivencia entre los pacientes que iniciaron tratamiento en dos periodos de tiempo, 1978-1989 y 1989-1994, se encontró que existía una mayor supervivencia entre los pacientes que comenzaron el TSFR en el último periodo considerado, sin embargo, estos resultados no alcanzaban significación estadística $(\mathrm{p}=0,44)$. La curva de supervivencia actuarial de los pacientes diabéticos en HD (90\%, 73\% y 56\%, al año, 3 años y 5 años respectivamente) era discretamente mejor que la de los pacientes en DPCA (93, 67 y $51 \%$ al año, 3 años y 5 años respectivamente), esta diferencia no fue significativa. Hay que tener en cuenta, que los pacientes en DPCA eran significativamente más jóvenes y con una mayor proporción de diabetes tipo 1 que los de HD.

Mediante análisis de regresión múltiple de Cox, utilizando como covariantes la edad al inicio del tratamiento, sexo, tipo de diabetes, tratamiento sustitutivo inicial de la función renal, el haberse trasplantado, existencia de retinopatía diabética, la presencia o no de cardiopatía, ACVA o isquemia en los miembros inferiores, sólo encontramos como factores predictivos de muerte independientes, el trasplante, que aparecía como factor de buen pronóstico y la isquemia en los miembros inferiores, como factor de mal pronóstico. Excluyendo la variable trasplante, la edad adquiría un valor pronóstico independiente.

\section{DISCUSIÓN}

La diabetes mellitus se ha convertido en la causa más frecuente de insuficiencia renal terminal, por delante de las glomerulonefritis y nefropatías tubulointersticiales y de hecho, en nuestra área sanitaria representa, en la actualidad, el 30\% de todas las causas. Hemos asistido a un incremento en el porcentaje de pacientes diabéticos en tratamiento sustitutivo de la función renal (5-8). En nuestra área sanitaria, desde 1983 a 1998, la incidencia de pacientes diabéticos incluidos en TSFR por año y millón de población, se ha triplicado. Este incre- 
mento se ha producido a costa de los diabéticos tipo 2 , que han pasado de representar el $17 \%$ de los diabéticos incluidos en TSFR a un $81 \%$, con un cambio en su incidencia de 1,7 a 25,9 por mp. Por el contrario, la incidencia de diabéticos tipo 1 incluidos en TSFR ha disminuido discretamente, de 8,3 a $6,1 \mathrm{pmp}$. De forma paralela se ha asociado un aumento, en 17 años, de la edad media con la que estos pacientes comienzan TSFR. Los resultados obtenidos con nuestros pacientes son totalmente concordantes con los de otros autores $(5-8,10)$. En Estados Unidos, en 1994, la incidencia de diabéticos que iniciaron TSFR fue de $70 \mathrm{pmp}$, incluida en una incidencia total de 198 pmp, 35,3\% de diabéticos respecto al total, datos muy superiores a los europeos. La mayoría de estos pacientes eran diabéticos tipo 2 (18). Quince años antes esa misma incidencia era de 21 pmp (19). En Europa, el porcentaje de diabéticos del total de pacientes que iniciaban TSFR era del 3\% en 1976 , $11 \%$ en 1985 y del $17 \%$ en 1992 , con unas diferencias muy marcadas entre unos países y otros: 21 diabéticos por mp en Austria, 14 pmp en Dinamarca, 10 pmp en Francia y 8,5 pmp en Holanda (4,9). En 1993, en la región de Neckar en Alemania, se realizó un estudio epidemiológico sobre este problema. La incidencia fue de $52 \mathrm{pmp}$, siendo un $90 \%$ diabéticos tipo 2 (10). En este estudio observaron que muchos diabéticos tipo 2 , fallecían antes de desarrollar IRT.

¿Cuál es la causa o causas de este incremento en la incidencia de diabéticos en IRT? La causa es probablemente multifactorial. En primer lugar, porque la prevalencia de la diabetes tipo 2 en la población general está aumentando, en parte por que la sociedad está envejeciendo, en parte porque el nivel económico en los países desarrollados está mejorando y el estilo de vida es más sedentario (11). En segundo lugar, el mejor control y tratamiento de los diabéticos ha permitido que muchos de ellos lleguen a una situación de insuficiencia renal terminal antes de fallecer por otras causas. Por otro lado, se están empleando unos criterios de inclusión en diálisis más amplios, que afectan a pacientes cada vez más mayores y con más pluripatología. Las diferencias genéticas, étnicas, nutricionales y de forma de vida pueden parcialmente explicar el incremento de la incidencia y sus diferencias geográficas, pero estos factores no cambian en 10 años suficientemente como para explicar las grandes diferencias entre áreas geográficas vecinas y el incremento objetivado. El diferente desarrollo sanitario y el criterio médico deben jugar un papel importante en esta cuestión. De ser esto así, las grandes diferencias encontradas irán disminuyendo en los próximos años, aumentando el número de diabéticos tipo 2 en TSFR, creando un verdadero problema epidemiológico-sanitario, por su coste social y económico.

Los diabéticos en TSFR tienen peor pronóstico que los pacientes con otras causas de insuficiencia renal y depende en gran medida de la afectación sistémica que presentan. Este peor pronóstico, aparece en todas las modalidades de TSFR: HD $(10,20)$; DPCA $(21,22)$ y trasplante renal $(9,10)$. Los pacientes diabéticos no sólo tienen una mortalidad mayor sino que además, su morbilidad es muy alta. En nuestra serie se observa una supervivencia global semejante a la de algunos grupos como el de Newcastle (23) y superior en un $10-20 \%$ respecto a la de otros $(9,10,24,25)$. Estos resultados se pueden deber a una proporción mayor de pacientes trasplantados y menor en diálisis peritoneal.

El concepto de "TSFR integrado" es muy importante para los diabéticos. Estos pacientes precisan cambiar de modalidad terapéutica con frecuencia. El tratamiento precoz de las complicaciones clínicas y una política preventiva de las complicaciones del acceso vascular tienen también un gran valor (26). En nuestra serie, el paso de los años, la mayor experiencia y las mejores técnicas de diálisis no han logrado mejorar significativamente la supervivencia. Esto probablemente se explique porque los pacientes diabéticos tipo 2 que incluimos actualmente en diálisis, presentan una comorbilidad mucho mayor y de ahí que los resultados esperados con las mejoras técnicas y asistenciales queden contrarrestados por un mayor riesgo de mortalidad.

En nuestro Hospital, las características del tratamiento con hemodiálisis en estos pacientes en nuestro hospital son: líquido de diálisis con $138 \mathrm{mmol} / \mathrm{L}$ de Na y $39 \mathrm{mmol} / \mathrm{L}$ de bicarbonato, $70 \%$ de los pacientes con dializadores de alta permeabilidad y biocompatibilidad; $40 \%$ en técnica de AFB "Acetate Free Biofiltration"; Kt/V mayor de 1,2 (Sargent, Gohst) o 1,35 (Daugirdas II); duración de las diálisis entre 3 y 4 horas por sesión y lo más importante, la consecución de una buena tolerancia a la técnica. En los diabéticos, la AFB permite un control mejor del metabolismo ácido-base, del estado nutricional y de la tolerancia, que la hemodiálisis con bicarbonato (27).

La micro y macroangiopatía permanecen como el mayor problema clínico en los diabéticos en TSFR, pues son los principales condicionantes de su morbilidad y mortalidad. En nuestra experiencia, la angiopatía distal es el factor independiente de mortalidad con mayor peso en los diabéticos en TSFR. Sí es necesario desarrollar nuevas estrategias de prevención de la angiopatía y nefropatía en los pacientes diabéticos.

\section{Bibliografía}

1. Spanish Nephrology Association Registry Committee, Regional Registries and Pediatric Registry. 1997 Spanish Nephrology Association (Sociedad Española de Nefrología) Report on Dialysis and Transplantation. Nephrol Dial Transplant 1999; 14: 2841-2845.

2. USRDS. II. Incidence and prevalence of ESRD. Am J Kid Dis 1998; 32, suppl. 1: s38-s49.

3. Renal Data System. USRDS 1996. Annual Data Report. National Institutes of Health, National Institute of Diabetes and Digestive and Kidney Diseases. Bethesda, MD, 1997.

4. Berthoux F, Jones E, Gellert R, Mendel S, Saker L, Briggs D with the participation of the National registries. Epidemiological data of treated end-stage renal failure in the European Union during the year 1995:

report of the European Renal Association Registry and the National Registries. Nephrol Dial Transplant 1999; 14: 2332-2342.

5. Abaigar et al. Grupo de estudio de la Sociedad Nefrológica del Norte. Tratamiento de sustitucion en la nefropatía diabética. Nefrología 1992; 12: 121-127.

6. Pérez-García R, DallÁnesse C, Jofre R, López Gómez JM, Junco E, Gómez Campdera F, Verde E, Valderrabano F. Tratamiento sustitutivo de la función renal en diabéticos: diecisiete años de experiencia. Nefrología 1996; 16: 52-58.

7. Rychlik I, Miltenberger-Miltenyi G, Ritz E. The drama of the continuous increase in end-stage renal failure in patients with type II diabetes mellitus. Nephrol Dial Transplant 1998; 13 (Suppl 8): 6-10. 
8. Rodríguez JA, Cleries M, Vela E and renal Registry Committee. Diabetics patients on renal replacement therapy: analysis of Catalonia. Nephrol Dial Transplant 1997; 12: 2501-2509.

9. Brunner FP, Selwood NH. Profile of patients on RRT in Europe and death rates due to major causes of death groups. EDTA Registry. Kidney Int 1992; 42 (S38): S4-S15.

10. Lippert J, Ritz E, Schwarzbeck A, Schneider P. The rising tide of endstage failure from diabetic nephropathy type II: an epidemiological analysis. Nephrol Dial Transplant 1995; 10: 462-467.

11. Ruggenenti P, Remuzzi G. Nephropathy of type-2 diabetes mellitus. J Am Soc Nephrol 1998; 9: 2157-2169.

12. Khanna R. Peritoneal dialysis in diabetic end-stage renal disease patients. En: Twardowski ZJ, Nolph KD, Khanna R. eds. 22 Contemporary Issues in Nephrology. peritoneal dialysis. Churchill Livingstone, NY 1990; 211-229.

13. Nolph K, Lindblad AS, Novak JW. Continuous ambulatory peritoneal dialysis. N Engl J Med 1988; 318: 1595-1599.

14. Koch M, Thomas B, Tschöpe W, Ritz E. Survival and predictors of death in dialysed diabetics. Diabetologia 1993; 36: 1113-1117.

15. Viglino G, Cancarini G.C, Catizone L, Cocchi R, De Vecchi A, Lupo A, Salomone M, Segoloni G.P, Giangrande A. Ten years experience of CAPD in diabetics: comparison of results with non-diabetics. Nephrol Dial Transplant 1994; 9: 1443-1448.

16. Catalano C, Marino C, Portorino M, Laicogino ANED. Comparison between survivalof diabetic of diabetic and nondiabetic patients treated by renal replacement therapy in Italy. Nephrol Dial Transplant 1994; 9: 952-956.

17. Hirschl MM. The patients with type II diabetes and uraemia - to transplant or not to transplant. Nephrol Dial Transplant 1995; 10: 1515-1516.

18. Renal Data System. USRDS 1994 annual data report. National Institutes of Health, National Institute of Diabetes and Digestive and Kidney
Diseases. Bethesda, MD, 1994.

19. Renal Data System. USRDS 1998 annual data report. Bethesda, MD: National Institutes of Health, National Institute of Diabetes and Digestive and Kidney Diseases. April 1998. (NIH publication $\mathrm{n}^{\circ}$ 98-3176).

20. Held PJ, Brunner FP, Odaka M, García JR, Port FK, Gaylin DS. Fiveyear survival for end-stage renal disease patients in the United States, Europe and Japan, 1982 to 1987. Am J Kidney Dis 1990; 15: 451-457.

21. Markell MS, Friedman EA. Diabetic nephropathy: management of the end-stage patients. Diabetes Care 1992; 15: 1266-1238.

22. Viglino G, Cancarini G.C, Catizone L, Cocchi R, De Vecchi A, Lupo A, Salomone M, Segoloni GP, Giangrande A. Ten years experience of CAPD in diabetics: comparison of results with non-diabetics. Nephrol Dial Transplant 1994; 9: 1443-1448.

23. Catalano C, Goodship THJ, Tapson JS, Venning MK, Taylor RMR, Proud G, Tunbridge WMG, Elliot W, Ward MK, Alberti KGMM, Wilkinson R. Renal replacement therapy for diabetic patients in Newcastle upon Tyne and the Nothern region, 1964-88. Br Med J 1990; 301: 535-540.

24. Friedman EA. Management choices in diabetic end-stage renal disease. Nephrol Dial Transplant 1995; 10 (Suppl 7): 61-69.

25. Romagnoli GF, Di Landro D, Catalano C, Goepel V, Milan Manani S, Ruffatti AM, Marchini F, Naso A. Short-term outcome of diabetic patients in renal replacement therapy. Nephrol Dial Transplant 1998; 13 (Suppl 8): 30-34.

26. Ridao N, Polo JR, Pérez-García R, Sánchez M, Réngel MA, Gómez Campderá FJ. Accesos vasculares para diálisis en el anciano. Nefrología 1998; 13, suppl. 4: 22-26.

27. Verzetti G, Navino C, Bolzani R, Galli G, Panzetta G. Acetate-free biofiltration versus bicarbonate haemodialysisi in the treatment of patients with diabetic nephropathy: a cross-over multicentric study. Nephrol Dial Transplant 1998; 13: 955-961. 